\title{
Hepatocarcinoma gigante unifocal en mujer con ingesta prolongada de anticonceptivos hormonales: ¿casualidad 0 causalidad?
}

\author{
Unifocal giant hepatocellular carcinoma prolonged intake of hormonal \\ contraceptives. Coincidence or Causality?
}

Victor Manuel Lopez Mouriño, José Lopez Castro, Benigno Monteagudo Sanchez, Enrique Jesús Alvarez Asensio

Servicio de Medicina interna. Hospital Comarcal de Monforte de Lemos

\begin{abstract}
Resumen
Presentamos un caso de una paciente de mediana edad con ingesta mantenida de anticonceptivos hormonales (AH) que desarrolla múltiples adenomas hepáticos (asociación bien documentada) y un carcinoma hepatocelular $(\mathrm{CHC})$ unifocal gigante, sin encontrar otros factores de riesgo para el mismo (enolismo, tabaquismo, cirrosis, hemocromatosis,...). Si bien en la literatura no hay unanimidad respecto a la asociación del consumo de $\mathrm{AH}$ con el $\mathrm{CHC}$, algunos autores ya contemplaron esa posibilidad, excluyendo también otras etiologías reconocidas de CHC, por lo que debería reevaluarse si su asociación es aleatoria o por el contrario existe nexo causal.
\end{abstract}

Palabras clave: carcinoma hepatocelular, anticonceptivos hormonales, etiología.

\section{Introducción}

El CHC es la neoplasia primaria hepática más frecuente. Constituye el sexto tumor más habitual en el mundo. Su frecuencia según la literatura oscila entre más de 500.000 y el millón de casos $^{2}$ diagnosticados al año y es la tercera causa más común de muerte por cáncer. La proporción entre varones y mujeres es de aproximadamente 4:1 (la proporción se iguala en países sin cirrosis, siendo la proporción 9:1 en muchos países de elevada incidencia de cirrosis). 2,3 El principal factor de riesgo es la hepatopatía crónica por virus $B$ y C. Otras etiologías contempladas en la literatura son otras causas de cirrosis, consumo de etanol durante largo tiempo, aflatoxinas u otras micotoxinas y ya inusuales cirrosis biliar primaria, déficit de alfa1antitripsina, enfermedades de depósito de glucógeno, porfiria cutánea tardía, tirosinemia hereditaria y enfermedad de Wilson. ${ }^{2,3}$

\section{Caso clínico}

Mujer de 46 años que presenta como únicos antecedentes relevantes cefaleas premenstruales, 1 aborto y 2 partos, así con consumo de AH (Drospirenona $3 \mathrm{mg}$,Etinilestradiol 0,03 mg) sin otros antecedentes relevantes durante 22 años.

Acude a consultas externas de Digestivo por síndrome general consistente en astenia, anorexia y pérdida de peso de $3 \mathrm{~kg}$, acompañada de incremento notable del perímetro abdominal, dispepsia y diarrea.

\begin{abstract}
We present a case of a middle-aged patient with sustained intake of hormonal contraceptives (AH) which develops multiple hepatic adenomas (well documented association) and hepatocellular carcinoma (HCC) unifocal giant without finding other risk factors for it (alcoholism, smoking, cirrhosis, hemochromatosis,...). While there is no unanimity regarding the association of consumption of $\mathrm{AH}$ with $\mathrm{CHC}$ in the literature, some authors already contemplated this possibility, also excluding other recognized etiologies of HCC, so you should assess whether your association is random or else there is a causal link.
\end{abstract}

Keywords: hepatocellular carcinoma, oral contraceptives, etiology

A la exploración se objetiva un abdomen voluminoso de aspecto gestacional de 6 meses, comprobándose a la palpación la existencia de un proceso tumoral centrado en mesogastrio. En la analítica anemia normocítica con hb 11,2 g/dl, mínia elevación de bilirrubina (1,7 mg/dl), ligera hipertansaminemia (AST: 203 UI/L; ALT: 156 UI/L; GGT. :85 UI/L) , con elevación de marcadores tumorales (CA 125: 66. 9 U/ml; CA 19-9:116. 9 U/ml. AFP :200. 16 U/Ml). Cobalaminas (Vitamina B 12). Resto incluido perfil hepático serologías vírus $B, C$, crioglobulinas, ANA, haptoglobina, cobre, ceruloplasmina, alfa1antitripsina,... normales o negativos.

En el TAC abdómino pélvico se describe una masa hepática gigante de $19 \times 14 \times 23 \mathrm{~cm}$ (diámetros transverso, AP y CC). Heterogénea, aparentemente encapsulada y con efecto de masa-desplazamiento de estructuras vasculares hepáticas. Muestra realce periférico y de tabiques intralesionales, zonas de probable degeneración hemorrágica o necrótica e importante vascularización en fase arterial, afectando a la totalidad del LHD, salvo segmento VII y también a los segmentos mediales del LHI compatibles con adenomas. Se aprecian también hemangiomas en el segmento VII $(32 \mathrm{~mm})$ y segmento VIII $(32 \mathrm{~mm})$ y al menos dos lesiones hipervasculares de $19 \mathrm{~mm}$ (segmento VII) y $12 \mathrm{~mm}$ (segmento II). Se insinúan pequeñas lesiones también hipervasculares de muy pequeño tamaño en el LHI compatibles con adenomas, ya que lavan en fase portal. El Páncreas, estómago y vesícula biliar se encuentran desplazados lateralmente por la masa sin objetivarse otras anomalías.

Se realizó PAAF guiada por TAC de dicha masa, siendo el diagnóstico anatomopatológico de $\mathrm{CHC}$, se realizó PET que no evidenció enfermedad metastática. Tras evaluación del comité de tumores se 


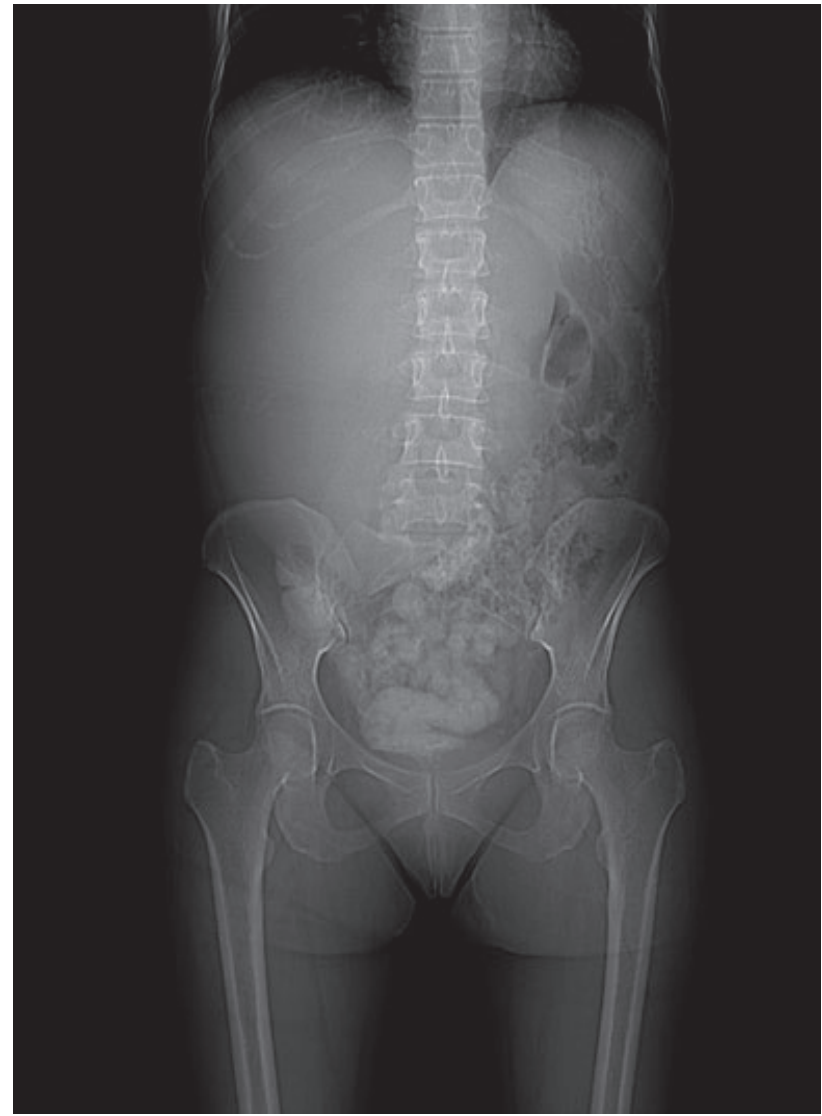

remitió al hospital de referencia para valoración de trasplante que fue desestimado. Se realizó arteriografía diagnóstica incluyendo estudios con cateterización selectiva del tronco celiaco y la arteria mesentérica superior apreciándose gran masa hipervascularizada con múltiples aportes arteriales dependientes de ramas de la arterias hepática y mesentérica superior. Se procece a cateterizar selectivamente la arteria hepática común y realizamos inyección de endoesferas 300-500 $\mu \mathrm{m}$ cargadas con Adriamicina. Posteriormente se inyectan endoesferas 900 um para embolización física. Un mes después se intentó nueva embolización, pero el hallazgo de venas ectásicas que drenan directamente en suprahepáticas, no llegando a visualizarse en ningún momento la vena porta, contraindicó el tratamiento endovascular, dado el alto riesgo de paso de micropartículas a cavidades cardiacas derechas.

La paciente actualmente está en seguimiento en consultas externas de Oncología, manteniéndose tratamiento con dexametasona $4 \mathrm{mg} / \mathrm{d}$ y desloratadina $10 \mathrm{mg} /$ día.

\section{Discusión}

El principal factor de riesgo para el desarrollo de $\mathrm{CHC}$ es el VHB,asociado o no a aflatoxina. Entre el 10 y el 30\% de los $\mathrm{CHC}$ relacionados con VHB se desarrollan sobre hígado no cirrótico. En pacientes infectados por el VHB se ha establecido que la carga viral y la presencia de los antígenos HBe y HBs son factores de riesgo, independientes de la cirrosis, para la aparición de este tumor. ${ }^{3}$ Esta enfermedad afecta de manera preferente a pacientes con hepatopatía crónica que han desarrollado cirrosis, especialmente la relacionada

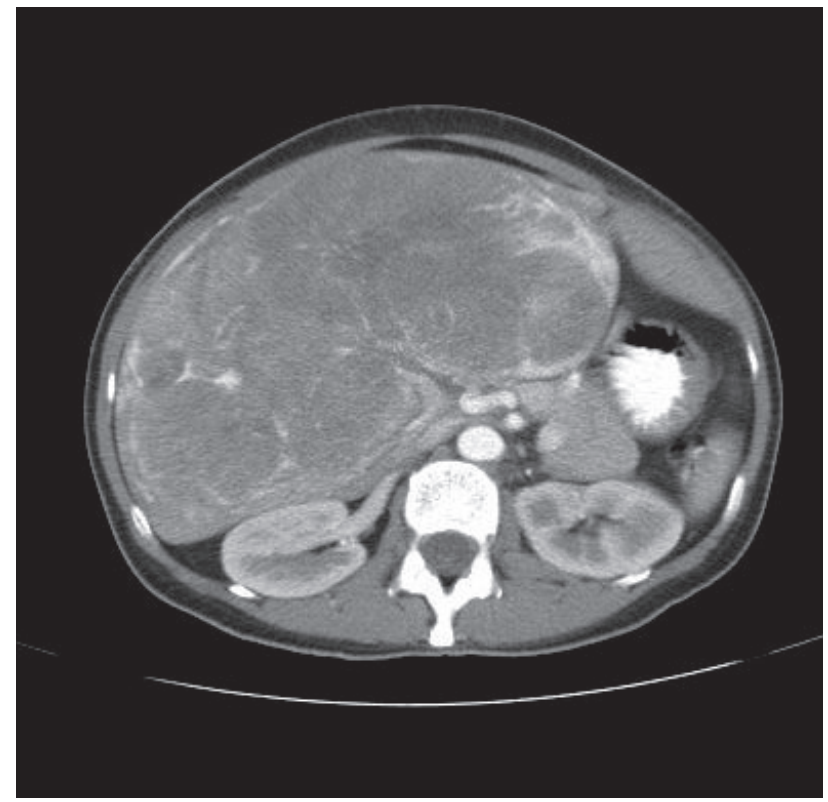

con los virus de la hepatitis B y C, siendo en este grupo de sujetos el CHC la causa más frecuente de muerte. ${ }^{1}$

Otras posibles causas contempladas en la literatura son otras causas de cirrosis, consumo de etanol durante largo tiempo, aflatoxinas u otras micotoxinas y ya inusuales cirrosis biliar primaria, déficit de alfa1 antitripsina, enfermedades de depósito de glucógeno, porfiria cutánea tarda, tirosinemia hereditaria y enfermedad de Wilson. No se sabe si la propia cirrosis es un factor predisponente para $\mathrm{CHC}$ o si las causas primarias de la cirrosis en realidad constituyen los factores carcinógenos. Sin embargo, cerca de $20 \%$ de los estadounidenses con $\mathrm{CHC}$ carece de cirrosis primaria. Las patologías que se asocian al "síndrome metabólico"(obesidad, diabetes mellitus, dislipemia, resistencia a la insulina)se asocian a una mayor mortalidad relacionada con el $\mathrm{CHC} .^{8,4}$

El tabaco también incrementa el riesgo de $\mathrm{CHC}^{5}$. Algunos autores si recogen como posible causa la toma de anaovulatorios orales durante largo tiempo.,7

La AH que tomaba nuestras enferma estaba compuesta de Drospirenona y el Etinilestradiol. La Drospirenona, también conocida como 1,2-dihidrospirorenona, es una progestina esteroidea anti-androgénica sintética análoga a la espironolactona usada en algunas formulaciones de píldoras anticonceptivas así como y píldoras de terapia de reemplazo hormonal posmenopáusico. Difiere de otras progestinas sintéticas en su más cercano parecido a la progesterona natural. El Etinilestradiol que es un estrógeno derivado del estradiol, fue el primer análogo del estrógeno sintetizado en Berlín en 1938, y es uno de los medicamentos más usados en el mundo.

Nuestra paciente padece múltiples adenomas hepáticos que se relacionan con su consumo prolongado de $\mathrm{AH}$, sin embargo no encontramos ningún factor etiológico para el CHC (habiéndose descartado los factores de riesgo habitua- 
les para el mismo) por lo que consideramos que pudiera también estar relacionado con el consumo durante años de dicha AH. Esta posible asociación suscitó controversia en la literatura ya que, aunque mayoritariamente algunos autores rechazan dicha asociación, también es cierto que otros ya contemplan tal posibilidad.

Ye MQ et al ${ }^{8}$ publican un caso similar de una mujer de 42 años que consumió durante 25 años anticonceptivos orales, en la que se encontraron dos masas hepáticas,un adenoma hapatocelular y un carcinoma, lo cual podría sugerir que inicialmente ambas lesiones eran adenomas, evolucionando posteriormente una de ellas a hepatocarcinoma. Mansvelt B et al presentan otro caso de $\mathrm{CHC}$ en una mujer de 36 años que tomó anticonceptivos durante 18 años $^{9}$. Fiel et al presentan 4 casos de mujeres que presentaron hepatocarcinomas tras el uso durante largo tiempo de contracepción hormonal (anticonceptivos-androgénicos anabólicos), ninguno de los cuales presentaba secuencias virales, lo cual fortalece el posible papel de los $\mathrm{AH}$ en hepatocarcinogenesis ${ }^{10}$. En contraposición, algunos autores afirman que no existe dicha asociación, como por ejemplo Rabe T et al. en 1995

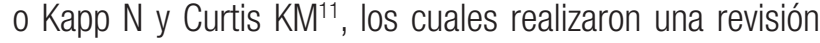
bibliográfica ( PubMed y Cochrane), no encontrando relación entre los tumorres hepáticos y el consumo de $\mathrm{AH}$. Por otra parte, McGlynn KA et al, en una suma de 11 cohortes, que abarcaban casi 800. 000 mujeres, concluyeron que no hubo asociación entre el uso de anticonceptivos orales y $\mathrm{CHC}{ }^{12}$

\section{Bibliografía}

1. Pin Vieito N, Guerrero Montañés A, Delgado Blanco M. Hepatocarcinoma: estado actual. Galicia Clin 2014; 75 (4): 171-181

2. Brian I. Carr. Tumores de hígado y vías biliares. Harrison Príncipios de Medicina Interna $17^{\mathrm{a}}$ edicción 580-585.

3 Bosch FX, Ribes J, Diaz M, Cleries R. Primary liver cancer: Worldwide incidence and trends. Gastroenterology. 2004; 127 (Suppl 1):S5-S16.

4 Yasui K, Hashimoto E, Komorizono Y, Koike K, Arii S, Imai Yet al. Characteristics of patients with nonalcoholic steatohepatitis who develop hepatocellular carcinoma. Clin Gastroenterol Hepatol. 2011; 9: 428 - 33

5 Kuper H, Tzonou A, Kaklamani E, Hsieh CC, Lagiou P. Adami HO, et al. Tobacco smoking, alcohol consumption and their interaction in the causation of hepatocellular carcinoma. Int J Cancer. 2000; 85: 498-502.

6. Yu MC, Tong MJ, Govindarajan S, Henderson BE C. Nonviral risk factors for hepatocellular carcinoma in a low-risk population, the non-Asians of Los Angeles County, California. J Natl Cancer Inst. 1991 Dec 18:83(24):1820-6.

7 J Neuberger, D Forman, R Doll, R Williams. Oral contraceptives and hepatocellular carcinoma. Br Med J (Clin Res Ed). 1986 May 24; 292(6532): 1355-1357.

8. Ye MQ, Suriawinata A, Ben Haim M, Parsons R, Schwartz ME. A 42-year-old woman with liver masses and long-term use of oral contraceptives. Semin Liver Dis. 1999; 19(3):339-44

9 Mansvelt B, Etienne PY, Bertrand C, Henrion J, Gerard R. Hepatocellular carcinoma associated with precocious puberty and oral contraceptives. A case report. Acta Chir Belg. 2003 Aug;103(4):412-3.

10 Fiel Ml, Min A, Gerber MA, Faire B, Schwartz M, Thung SN. Hepatocellular carcinoma in long-term oral contraceptive use. Zentralbl Gynakol. 1995;117(3):153-6.

11 KappN, Kathryn M. Hormonal contraceptive use among women with liver tumors: a systematic review. Contraception 2009. October (4): 387-390

12. McGlynn KA, Sahasrabuddhe W, Campbell PT, Graubard BI, Chen J, Schwartz LM, Petrick JL, Alavanja MC, Andreotti G, Boggs DA, Buring JE, Chan AT, Freedman ND, Gapstur SM, Hollenbeck AR, Hou L, King LY, Koshiol J, Linet M, Palmer JR, Poynter JN, Purdue M, Robien K, Schairer C, Sesso HD, Sigurdson A, Wactawski-Wende J, Zeleniuch-Jacquotte A. Hormonal contraceptive use among women with liver tumors: a systematic review. Contraception 2009 October (4): 387-390. Br J Cancer. 2015 Mar 31;112(7):1266-72

\section{Normas de publicación Galicia Clínica}

Galicia Clínica es la revista oficial de la Sociedad Gallega de Medicina Interna (SOGAMI). Se publican 4 números al año, simultáneamente en papel y en www.galiciaclinica. info, incluyéndose en el segundo de ellos las comunicaciones enviadas a la correspondiente Reunión Ordinaria de la SOGAMI. GALLIA CLíNICA evaluará para su publicación trabajos médicos relacionados preferentemente con la Medicina Interna
suS subespecialidades, y/o con problemas médicos prevalentes en la Comunidad Autónoma de Galicia. Se admitirán para evaluación trabajios en castellano, gallego, inglés y portugués.

Para el envio de originales se ha habilitado un formulario en la pagina web www.galiciaclinica.info. El sistema confirmarála entrega y permitirá consultar el estado del manuscrito. No se aceptarán originales enviados por otros métodos.

Fcomité ella contite editónal, eventualmente con la ayuda de revisores extemos, evaluarál los trabajos enviados decidiendo si procéde autor las pruebas de imprenta para la corrección de posibles erratas.

Ls trabajos reunińn los requisitos de uniformidad habtuales en revistas biomédicas Dichos requistos se pueden consuttar en "Uniform Requirements for Manuscripts Submitted to Biomedica Jurmals. Writing and Editing for Biomedice Publication. Updated April 2010", disponible en hitp://mmwicmie org. Se recomienda encarecidamente leer en especial la sección "Preparing a Manuscript for Submission to a Biomedical Journal" (http://mww.icmje. org/manuscript 1 prepare. $\mathrm{html)}$ y seguir fielmente sus indicaciones a la hora de redactar el trabajo a enviar.

Se recomienda el empleo de los programas más habituales de edición de texto (EE.. Word) tanto para el texto como para las tablas.

Dado que la mayoría de las páginas se imprimen en blanco y negro, se aconseja evitar en tablas y figuras en la medida de lo posible el uso de colores o tramas que no tengan el adecuado contraste para su identificación.

Las figuras o imágenes se enviarán en archivo aparte, como archivo de imagen (jpeg o similar) o como PDF con una resolución de 300 ppp. a tamaño de impresión definitivo.

La revista presenta las siguientes secciones:

Editoriales

Habitualmente encargados por la dirección de la revista. Su extensión máxima será de 8 páginas de 30 líneas y se admitira una figura 0 una tabla y quince citas bibliográficas.

El número máximo de firmantes será de dos.

Originales

Trabajos de investigación sobre cualquier aspecto médico. La estructura general de los trabajos será la tradicional: Título: en el idioma original y en inglés, Resumen y Abstract en inglés, Palabras clave y Key words (que deben corresponder a los medical subjets headings -MESH- del Index Medicus), Introducción, Material y métodos, Resultados, Discusión, Bibliografía La extensión máxima recomendada del texto es de 20 páginas de 30 lineas, a las que se podrán añadir 5 figuras y 5 tablas, y un máximo de 30 citas bibliográficas.

El número máximo de firmantes será de ocho.

Originales breves

Trabajos de investigación que por sus características no precisan un mayor espacio. Estructura similar a la de los origina政 10 páginas de 30 líneas, 3 figuras, 3 tablas y 20 citas bibliográficas. El número máximo de firmantes será de seis.

\section{Revisiones}

Habitualmente encargadas por la dirección de la revista. La extensión máxima recomendada del texto es de 30 páginas de 列

El número máximo de firmantes será de tres.

Preguntas clínicas

En esta sección se tratará de responder de forma concreta y sucinta a preguntas clinicas concretas que, bien han motivado una controversia, o cuestionan actitudes arraigadas en la práctica diaria. La extensión máxima será de 6 páginas de 30 líneas, dos figuras y dos tablas y 15 citas bibliográficas.

El número máximo de firmantes será de dos.

Notas clínicas

Descripción de casos clínicos de excepcional interés. Constarán de una breve introducción, caso clínico, y discusión correspondiente. Su extensión máxima será de 6 páginas, 2 figuras y dos tablas y 15 citas bibliográficas.

El número máximo de firmantes será de cuatro.

Cartas al director

Comentarios, opiniones u observaciones sobre los diversos trabajos publicados con anterioridad en la revista. La extensión máxima será de 4 páginas de 30 líneas y se admitirá una figura o una tabla y diez citas bibliográficas.

El número máximo de firmantes será de dos.

Imágenes médicas

Imágenes curiosas, insólitas o demostrativas. Se acompañarán con un texto breve, como máximo 1 página de 30 líneas, en el que se explique el caso clínico, con una breve discusión acerca de la importancia de la imagen.

El número máximo de firmantes será de dos.

Resúmenes de Tesis doctorales

Elaborados por el autor, describirán el trabajo realizado; su extensión máxima será de 2 páginas de 30 líneas. Debe incluirse un apéndice con los datos correspondientes a Universidad, departamento, director de la tesis y fecha de presentación. Otros

a dirección de la revista considerará para su publicación cualquier artículo relacionado con la medicina en cualquier aspecto, aunque no se incliva exactamente dentro de los supuestos anteriores. En este caso se recomienda antes de su envio contactar con la dirección para acordar las características del mismo.

En el caso de que los trabajos enviados incluyan imágenes, figuras, tablas o textos sometidos a copyright, será responsabilidad de los autores la obtención de los permisos necesarios para su publicación.

Todas las opiniones o afirmaciones expresadas en los artículos corresponden a los autores de los mismos. Tanto el comité editorial como la SOGAMI declinan cualquier responsabilidad a este respecto.

Los trabajos publicados serán propiedad de GALCIA CLIINICA, cediendo los autores todos los derechos a la misma. 